\title{
Telomeric Repeat-Containing RNA
}

National Cancer Institute

\section{Source}

National Cancer Institute. Telomeric Repeat-Containing RNA. NCI Thesaurus. Code C116964.

A large, non-coding RNA that can be encoded by any chromosomal telomeric DNA sequence, and is an integral part of the telomeric heterochromatin. These oligoribonucleotides may play a role in the regulation of both telomere replication and chromosomal remodeling. Their sizes are dependent on the telomere sequence length. 